\title{
Impacto do álcool sobre os parâmetros da síndrome metabólica em adultos: uma revisão integrativa
}

\author{
Alcohol impact on the parameters of metabolic syndrome in adults: an integrative review \\ Impacto del alcohol sobre los parámetros del síndrome metabólico en adultos: una \\ revisión integrativa
}

Andréia Klier ${ }^{1 \star}$, Flora Miranda Arcanjo ${ }^{1}$, Isabel Fernandes de Souza ${ }^{1}$.

\begin{abstract}
RESUMO
Objetivo: Identificar as evidências científicas dos impactos, positivos ou negativos, do consumo do álcool nos parâmetros da Síndrome Metabólica. Métodos: Uma revisão integrativa da literatura, indexada nas bases Scientific Eletronic Library Online (SciELO), Medical Literature Analysis and Retrieval System Online (MEDLINE) e Literatura Latinoamericana y del Caribe en Ciencias de la Salud (LILACS), publicadas entre janeiro de 2009 e dezembro de 2019. Foram incluídos artigos originais, nos idiomas português, inglês e espanhol, com amostra $n>100$, faixa etária entre 18 e 61 anos, alinhados com a temática de pesquisa. Resultados: Esta revisão integrativa incluiu 14 estudos observacionais que abordavam os parâmetros metabólicos de composição corporal, perfil lipídico, pressão arterial e de metabolismo glicêmico. Os resultados evidenciados nos estudos foram analisados de acordo com o sexo, pois percebeu-se que os impactos do álcool foram divergentes quanto ao gênero dos participantes. Considerações Finais: Os impactos do álcool no índice de massa corporal, circunferência da cintura, pressão arterial e metabolismo glicêmico, não foram conclusivos para homens e mulheres. A única associação evidenciada está relacionada ao aumento dos valores de HDL-Colesterol e triglicerídeos, em ambos os sexos, em qualquer quantidade de álcool ingerida.
\end{abstract}

Palavras-chave: Etanol, Consumo de bebidas alcoólicas, Síndrome metabólica.

\section{ABSTRACT}

Objective: To identify scientific evidences of positive and negative impacts of alcohol consumption on the parameters of Metabolic Syndrome. Methods: An integrative theoretical review based on literature indexed in the following bases: Scientific Eletronic Library Online (SciELO), Medical Literature Analysis and Retrieval System Online (MEDLINE) and Literatura Latinoamericana y del Caribe en Ciencias de la Salud (LILACS), published between January 2009 and December 2019. Original articles in Portuguese, English and Spanish were included, with a sample $n>100$ (ages 18-61), aligned with the researched theme. Results: The previously mentioned integrative review includes 14 observational studies that focused on the metabolic parameters of body composition, lipid profile, blood pressure and glycemic metabolism. The evidenced results were analyzed according to gender, because the alcohol impact differs from men to women. Final Considerations: The alcohol impacts on the body mass index, abdominal circumference, blood pressure and glycemic metabolism were not conclusive for either men or women. The only evidenced association has to do whit an increase of HDL Cholesterol and triglyceride for both men and women, no matter the consumed amount of alcohol.

Keywords: Ethanol, Alcohol drinking, Metabolic syndrome.

\section{RESUMEN}

Objetivo: Identificar las evidencias científicas acerca de los impactos, positivos o negativos, del consumo de alcohol sobre los parámetros del Síndrome Metabólico. Métodos: Una revisión teórica integrativa de la literatura indexada en las bases de datos Scientific Eletronic Library Online (SciELO), Medical Literature Analysis and Retrieval System Online (MEDLINE) y Literatura Latinoamericana y del Caribe en Ciencias de la Salud (LILACS), publicada entre enero de 2009 y diciembre de 2019. Se incluyeron artículos originales, en los idiomas portugués, inglés y español, con muestras $n>100$, edad entre 18 y 61 años, alineados con la temática de investigación. Resultados: Esta revisión integrativa incluyó 14 estudios observacionales que abordaron los parámetros metabólicos de composición corporal, perfil lipídico, presión arterial y de metabolismo glucémico. Los resultados evidenciados en los estudios se analizaron según el sexo, pues se percibió que los impactos del alcohol eran divergentes en cuanto al género de los participantes.

${ }^{1}$ Centro Universitário União das Américas (UniAmérica), Foz do Iguaçu - PR.

*E-mail: deiaklier@yahoo.com.br

SUBMETIDO EM: 11/2020

| ACEITO EM: $12 / 2020$

PUBLICADO EM: $2 / 2020$ 
Consideraciones Finales: Los impactos del alcohol en el índice de masa corporal, la circunferencia de la cintura, presión arterial y el metabolismo glucémico, no son concluyentes para hombres y mujeres. La única asociación evidenciada se relaciona al aumento de los valores de HDL-Colesterol y triglicéridos, en ambos sexos, en cualquier cantidad de alcohol ingerido.

Palabras clave: Etanol, Consumo de bebidas alcohólicas, Síndrome metabólico.

\section{INTRODUÇÃO}

A Síndrome Metabólica (SM) pode ser caracterizada como uma série de anormalidades do metabolismo, que eleva o risco de desenvolvimento de doenças cardiovasculares (DCV) e Diabetes Mellitus tipo 2 (DM2) (XU H, et al., 2019). É considerada um problema de saúde pública grave em todo o mundo. Afeta mais de um bilhão de pessoas, e vem tendo aumento, de maneira progressiva, nas últimas três décadas (SAKLAYEN MG, 2018).

Identificada por Reaven, no final da década de 80, como "Síndrome X", as definições e formas de diagnosticar a SM modificaram-se a partir de atualizações ao longo do tempo e também de acordo com as organizações e entidades de saúde pública envolvidas (XU H, et al., 2019). No Brasil, a I Diretriz Brasileira de Diagnóstico e Tratamento da Síndrome Metabólica (I-DBSM) foi publicada em 2005, e encontra-se vigente, recomenda o uso da definição preconizada pelo National Cholesterol Education Program's Adult Treatment Panel III (NCEP-ATP III) (SOCIEDADE BRASILEIRA DE CARDIOLOGIA, 2005; NCEP-ATP III, 2001).

Desta forma, segundo a I-DBSM, os critérios utilizados como diagnóstico para SM são: circunferência da cintura (CC) $>88 \mathrm{~cm}$ para mulheres e $>102 \mathrm{~cm}$ para homens; glicemia em jejum $>110 \mathrm{mg} / \mathrm{dL}$; pressão arterial $\geq 130 \mathrm{mmHg} / \geq 85 \mathrm{mmHg}$; triglicerídeos $\geq 150 \mathrm{mg} / \mathrm{dL}$; HDL-C $<40 \mathrm{mg} / \mathrm{dL}$ para homens e $<50 \mathrm{mg} / \mathrm{dL}$ para mulheres. Para que o diagnóstico seja positivo para SM, é necessário que o indivíduo se encaixe em três ou mais dos parâmetros citados, e que se apresentem de forma concomitante. Além disso, o uso de medicação para qualquer uma dessas comorbidades não altera o diagnóstico (SOCIEDADE BRASILEIRA DE CARDIOLOGIA, 2005).

O desenvolvimento da SM, bem como de cada um de seus componentes, está diretamente associado aos fatores de risco modificáveis, tais como os hábitos de vida e os hábitos alimentares (AHIMA RS, 2016; RAMIRES EKNM, et al., 2018). Dentre os hábitos modificáveis que mais impactam o surgimento de doenças crônicas ou agudas, está o consumo de álcool. Considerado um hábito social, os índices de etilismo vêm aumentando a cada ano que passa (MANTHEY J, 2019).

A Organização Mundial da Saúde (OMS) alerta, há mais de uma década, que o consumo de álcool em qualquer quantidade pode trazer diversos riscos à saúde das pessoas, porém, os riscos aumentam quando 0 consumo for maior que 2 doses por dia ou se o indivíduo não se abstiver pelo menos dois dias na semana (HUMENIUK RE, et al., 2010).

No que se refere à dosagem, o National Institute on Alcohol Abuse and Alcoholism (NIAAA) complementa, afirmando que há distinção na tolerância ao consumo alcoólico entre homens e mulheres, recomendando que homens não ultrapassem 4 doses diárias, com limite de 14 doses semanais e que as mulheres não ultrapassem 3 doses diárias com limite de 7 doses semanais (ARCR EDITORIAL STAFF, 2018).

Assim, a OMS e o NIAAA, embora alinhados quanto ao risco presente, alertam para quantidades diferentes de álcool ingeridas. Por outro lado, há controvérsias em relação ao seu impacto sobre os componentes da $\mathrm{SM}$. Alguns estudos indicam que o consumo de álcool é capaz de reduzir a glicemia em jejum e o risco de desenvolvimento de DM2, enquanto outros indicam risco aumentado de obesidade, dislipidemia, hipertensão e hiperglicemia (ROH W, et al., 2009; DE JESUS SILVA AB, et al., 2011; TIMON R, et al., 2012; TERATANI T, et al., 2012; ROSOFF DB, et al., 2019).

Diante disso, a presente revisão integrativa buscou identificar as evidências presentes na literatura científica, no decênio compreendido entre janeiro de 2009 e dezembro de 2019, acerca dos impactos, positivos ou negativos, do álcool sobre os parâmetros da SM. Espera-se com esse manuscrito, contribuir a partir de comprovações científicas, sobre as condutas mais apropriadas na orientação quanto ao hábito etilista e sintomatologia de indicativos da presença de SM, para que os profissionais da saúde possam refletir a respeito do assunto. 


\section{MÉTODOS}

Trata-se de uma Revisão Integrativa da literatura, para a qual são necessários os seguintes passos: a identificação do tema a pesquisar e a elaboração da questão norteadora do estudo; a determinação dos critérios de inclusão ou exclusão de trabalhos; a identificação das estratégias de busca e bases de conhecimento a serem consultadas. Após a recuperação dos documentos, ainda há a identificação de quais foram os estudos pré-selecionados e selecionados, categorizando-os. Na sequência, pode-se, então, realizar a análise e interpretação dos dados para a síntese do conhecimento, no que se refere aos impactos do consumo alcoólico na SM (BOTELHO LLR, 2011).

Norteando este estudo, tem-se a questão: quais são as evidências presentes na literatura científica, publicada no decênio estabelecido entre janeiro de 2009 e dezembro de 2019, acerca dos impactos, positivos ou negativos, do álcool sobre os parâmetros da SM? A fim de respondê-la, realizou-se a busca de artigos indexados nas bases de dados eletrônicas LILACS, MEDLINE e SciELO.

Essas consultas ocorreram nos meses de agosto e setembro de 2020, utilizando-se os seguintes descritores, em língua portuguesa e inglesa: álcool, etanol, cerveja, vinho, síndrome metabólica, hipertensão arterial, hipertrigliceridemia, triglicerídeo, colesterol, hipercolesterolemia, obesidade, adiposidade abdominal, resistência à insulina, diabetes, hiperglicemia, adulto. Para compor as estratégias de recuperação dos documentos, fez-se o uso dos operadores lógicos "AND" e "OR", na presença dos sinônimos. Para maior alinhamento dos manuscritos recuperados ao tema desta revisão, foi necessário utilizar o operador "AND NOT" para os descritores, em língua portuguesa e inglesa: cirrose, esteatose hepática, doença hepática, psoríase, pancreatite, fator de risco.

Os descritores recuperaram 7.545 estudos. Posteriormente, com a aplicação da restrição do período de publicação entre 2009 e 2019, e em publicações nas línguas portuguesa, inglesa e espanhola, restaram 3.418 estudos potencialmente elegíveis para esta revisão. A partir desta identificação, ocorreu a primeira seleção de estudos através da leitura de todos os títulos e palavras-chave, buscando atender aos critérios de inclusão e exclusão. Nesta etapa foram incluídos apenas artigos originais, na língua portuguesa, inglesa ou espanhola. Foram excluídos livros, guias, teses, dissertações, monografias e artigos de revisão, bem como artigos desalinhados com a temática em questão. Teve-se o mesmo cuidado para não selecionar artigos duplicados. Ao final deste processo, restaram 90 manuscritos.

A segunda etapa de seleção, consistiu na leitura dos resumos dos 90 artigos pré-selecionados. Nesta fase, outros critérios de inclusão e exclusão foram observados. Incluíram-se apenas estudos com humanos e com população adulta compreendida na faixa etária entre 18 e 61 anos; e excluíram-se os estudos com animais ou plantas, com $n<100$ ou estudos que associavam o consumo de álcool ao de outros alimentos ou de outras substâncias ou ainda outros hábitos sociais. Desta forma, foram selecionados 14 artigos (Tabela 1).

Tabela 1 - Critérios de exclusão de estudos de acordo com cada base de dados.

\section{Critério}

Estudo inclui adolescentes e idosos em sua população

Estudo associa o consumo de álcool e outros alimentos

concomitantemente

Estudo focado na Educação Alimentar e Nutricional

Estudo que quantifica os efeitos da abstinência de álcool

Estudo associa o consumo de álcool ao tabaco

Estudo com $\mathrm{n}<100$

Estudo relacionado à genômica

Estudo relacionado a índices aterogênicos

Estudo relacionado a hábitos alimentares e de vida como um todo

Estudo que relaciona inversamente a patologia e o consumo de álcool

Estudo relacionado ao não diagnóstico da SM

Estudo relacionado ao uso de medicamentos

Estudo utilizando cerveja sem álcool

Fonte: Klier A, et al., 2020.
LILACS

$\begin{array}{ccc}0 & 0 & 33 \\ 0 & 0 & 1 \\ 1 & 0 & 2 \\ 0 & 0 & 1 \\ 1 & 0 & 2 \\ 0 & 0 & 3 \\ 0 & 0 & 1 \\ 1 & 0 & 4 \\ 8 & 9 & 4 \\ 0 & 0 & 2 \\ 1 & 0 & 0 \\ 1 & 0 & 0 \\ 1 & 0 & 0\end{array}$

Estes 14 artigos selecionados foram lidos na íntegra e compõem a presente Revisão Integrativa. É possível acompanhar o processo de seleção dos artigos através do fluxograma (Figura 1). 
Figura 1 - Identificação e seleção dos artigos sobre o impacto do álcool nos parâmetros da SM.

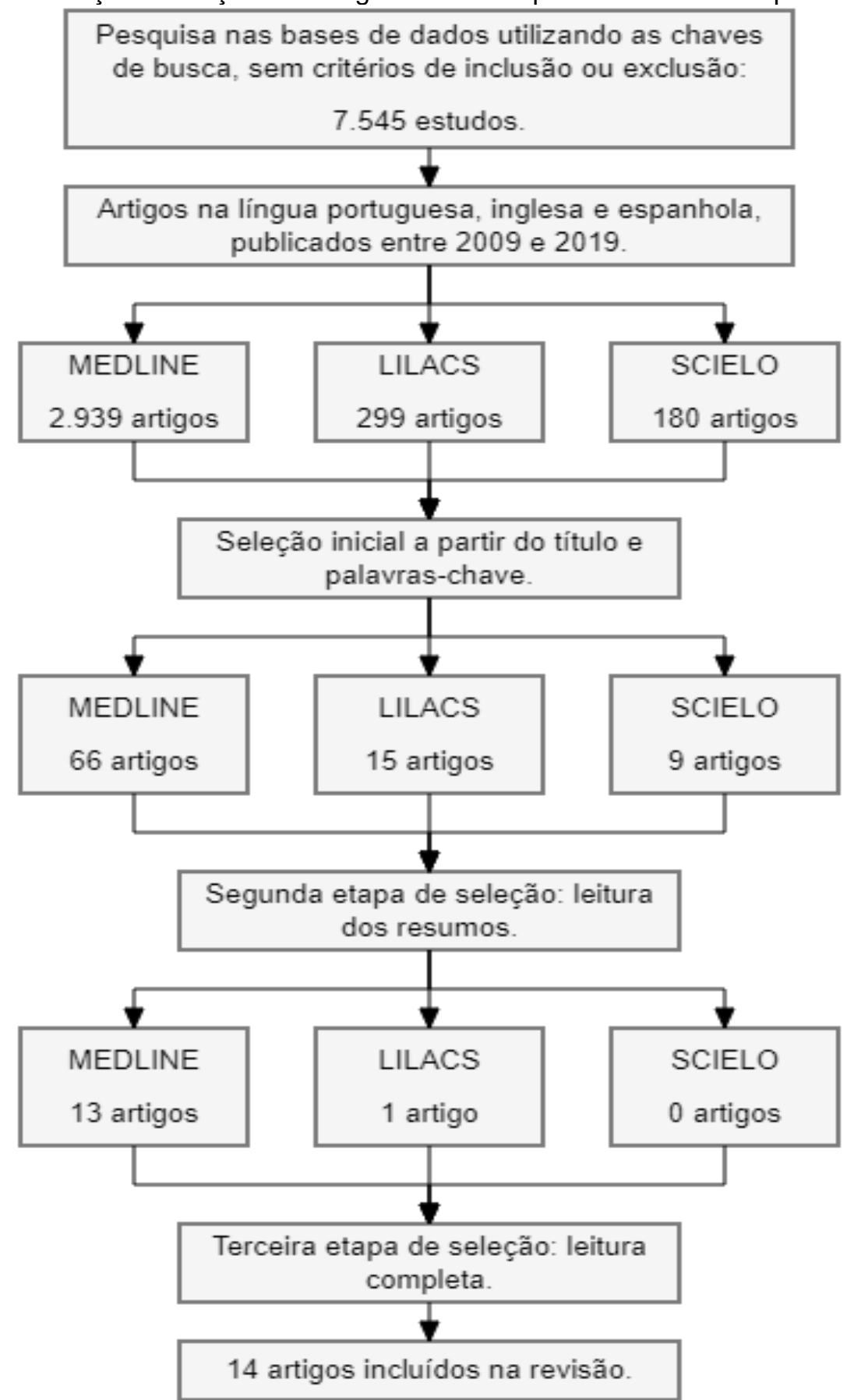

Fonte: Klier A, et al., 2020.

\section{RESULTADOS E DISCUSSÃO}

Os 14 artigos revisados caracterizam-se como estudos epidemiológicos observacionais de coorte ou transversais, com metodologias, objetivos e enfoques diferentes, assim como ocorrem em revisões integrativas. A seguir, serão detalhados estes artigos, destacando-se o objetivo de cada estudo e qual dos parâmetros da SM foi abordado no decorrer do mesmo (Quadro 1).

Os parâmetros da SM foram classificados em: composição corporal, avaliando a CC e também o IMC, visto que ambas as medidas estão intimamente associadas; perfil lipídico, enfatizando os resultados para TG e HDL-C; pressão arterial, distinguindo-se pressão arterial sistólica (PAS) e pressão arterial diastólica (PAD); e metabolismo glicêmico, observando-se a glicemia de jejum (GJ) e o risco de desenvolvimento de DM2, em que essa última é consequência da presença de alterações no metabolismo glicêmico. 


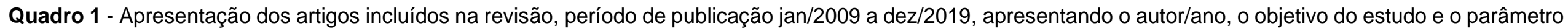
estudado.

\begin{tabular}{|c|c|c|c|c|c|}
\hline \multirow[b]{2}{*}{ Autor } & \multirow[b]{2}{*}{ Objetivo do estudo } & \multicolumn{4}{|c|}{ Parâmetro estudado } \\
\hline & & $\begin{array}{l}\text { Composição } \\
\text { corporal }\end{array}$ & $\begin{array}{c}\text { Perfil } \\
\text { lipídico }\end{array}$ & $\begin{array}{c}\text { Pressão } \\
\text { arterial }\end{array}$ & $\begin{array}{l}\text { Metabolismo } \\
\text { glicêmico }\end{array}$ \\
\hline ROSOFF DB, et al., 2019. & $\begin{array}{l}\text { Examinar as associações do consumo excessivo de álcool em alta } \\
\text { intensidade com os níveis de lipídios e enzimas da função hepática } \\
\text { em uma amostra transversal. }\end{array}$ & & $x$ & & \\
\hline ALBANI V, et al., 2018. & $\begin{array}{l}\text { Explorar a relação entre a energia obtida a partir de bebidas } \\
\text { alcoólicas e o IMC de homens e mulheres. }\end{array}$ & $x$ & & & \\
\hline TAYIE FA, et al., 2016. & $\begin{array}{l}\text { Investigar associações entre o consumo de bebidas alcoólicas e o } \\
\text { peso corporal. }\end{array}$ & $x$ & & & \\
\hline WELLMAN RJ, et al., 2016. & $\begin{array}{l}\text { Descrever a relação entre a frequência do consumo de álcool com a } \\
\text { PAS, em dois momentos, aos } 20 \text { e aos } 24 \text { anos. }\end{array}$ & & & $\mathrm{X}$ & \\
\hline ANEJA J, et al., 2013. & $\begin{array}{l}\text { Estudar a prevalência de SM e seus correlatos em homens } \\
\text { dependentes de álcool. }\end{array}$ & $\mathrm{X}$ & $x$ & $x$ & $x$ \\
\hline CHUNG HK, et al., 2013. & $\begin{array}{l}\text { Examinar as associações do consumo de álcool e a função das } \\
\text { células beta pancreáticas. }\end{array}$ & & $x$ & & $x$ \\
\hline BONNET F, et al., 2012. & $\begin{array}{l}\text { Investigar a relação entre o consumo de álcool com a resistência à } \\
\text { insulina, a secreção e a depuração de insulina e a concentração de } \\
\text { glucagon. }\end{array}$ & $\mathrm{X}$ & $X$ & $x$ & $x$ \\
\hline TERATANI T, et al., 2012. & $\begin{array}{l}\text { Examinar as relações dose-resposta entre o consumo de tabaco ou } \\
\text { álcool e o desenvolvimento da diabetes mellitus. }\end{array}$ & & & & $x$ \\
\hline BRANDHAGEN M, et al., 2012. & $\begin{array}{l}\text { Investigar a associação entre o consumo de álcool e } \\
\text { macronutrientes e a adiposidade, geral e central. }\end{array}$ & $X$ & & & \\
\hline WAKABAYASHI I., 2012. & $\begin{array}{l}\text { Determinar como a relação TG / HDL-C é influenciada pela ingestão } \\
\text { de álcool. }\end{array}$ & $x$ & $\mathrm{X}$ & & \\
\hline TIMON R, et al., 2012. & $\begin{array}{l}\text { Avaliar os efeitos do consumo excessivo nos finais de semana, sobre } \\
\text { diferentes parâmetros, em jovens universitários. }\end{array}$ & $\mathrm{X}$ & $x$ & $x$ & $x$ \\
\hline $\begin{array}{l}\text { DE JESUS SILVA AB, et al., } \\
\qquad 2011 .\end{array}$ & $\begin{array}{l}\text { Identificar a influência do consumo de bebidas alcoólicas na } \\
\text { adiposidade corporal de universitárias. }\end{array}$ & $x$ & & & \\
\hline ROH W. et al., 2009. & $\begin{array}{l}\text { Observar o desenvolvimento de GJ prejudicada ou DM2 de acordo } \\
\text { com a quantidade de álcool e IMC, durante } 4 \text { anos. }\end{array}$ & & & $X$ & $X$ \\
\hline ABRAMSON J, et al., 2009. & Investigar a relação entre o consumo de álcool e a pressão arterial. & & & $\mathrm{X}$ & \\
\hline
\end{tabular}

Fonte: Klier A, et al., 2020. 
Tabela 2 - Apresentação dos resultados dos artigos que estudaram a população masculina, incluindo autor, amostra e variáveis de consumo alcoólico.

\begin{tabular}{|c|c|c|c|c|c|c|}
\hline \multirow{2}{*}{ Autor } & \multirow{2}{*}{ Amostra } & \multirow{2}{*}{ Variáveis de consumo } & \multicolumn{4}{|c|}{ Parâmetro estudado } \\
\hline & & & Composição corporal & Perfil lipídico & Pressão arterial & Metabolismo glicêmico \\
\hline ROSOFF DB, et al., 2019. & 978 & $\begin{array}{c}<5 \text { doses/dia } \\
5 \text { a } 9 \text { doses/dia } \\
10 \text { a } 15 \text { doses/dia } \\
\geq 15 \text { doses/dia }\end{array}$ & 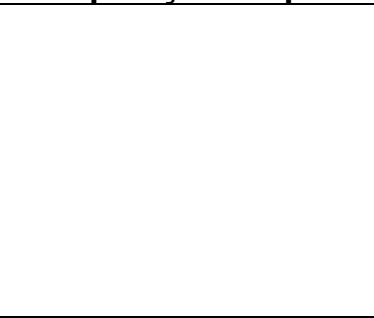 & $\begin{array}{c}{ }^{*} \mathrm{HDL}-\mathrm{C} \\
{ }^{*} \mathrm{TG} \\
{ }^{\uparrow} \mathrm{HDL}-\mathrm{H} \\
{ }^{\uparrow}{ }^{\uparrow} \mathrm{TG} \\
{ }^{\uparrow} \mathrm{HDL}-\mathrm{HD} \\
{ }^{\uparrow}{ }^{\uparrow} \mathrm{TG} \\
{ }^{\uparrow}{ }^{\uparrow} \mathrm{HDL}-\mathrm{C} \\
{ }^{\uparrow \uparrow} \mathrm{TG}\end{array}$ & & \\
\hline ALBANI V, et al., 2018. & 3.838 & $\begin{array}{c}0 \% \text { RDA } \\
>0-25 \% \text { RDA } \\
>25-50 \% \text { RDA } \\
>50-75 \% \text { RDA } \\
>75 \% \text { RDA }\end{array}$ & $\begin{array}{l}\text { * IMC } \\
+ \text { IMC } \\
+ \text { IMC } \\
+ \text { IMC } \\
{ }^{\uparrow} \text { IMC }\end{array}$ & & & \\
\hline TAYIE FA, et al., 2016. & 3.029 & $\begin{array}{c}0 \text { doses/dia } \\
1 \text { dose/dia } \\
2 \text { doses/dia } \\
3 \text { doses/dia } \\
\geq 4 \text { doses/dia }\end{array}$ & $\begin{array}{l}\text { * IMC } \\
\text { † IMC } \\
\uparrow \text { IMC } \\
\uparrow \text { IMC } \\
{ }^{\uparrow} \text { IMC }\end{array}$ & & & \\
\hline ANEJA J, et al., 2013. & 200 & $\begin{array}{c}\text { Não dependentes } \\
\text { Dependentes }\end{array}$ & $\begin{array}{l}{ }^{*} \mathrm{IMC} \\
{ }^{*} \mathrm{CC} \\
{ }^{+} \mathrm{IMC} \\
{ }^{+} \mathrm{CC}\end{array}$ & $\begin{array}{l}{ }^{*} \mathrm{HDL}-\mathrm{C} \\
{ }^{*} \mathrm{TG} \\
{ }^{\uparrow} \mathrm{HDL}-\mathrm{C} \\
\\
+\mathrm{TTG}\end{array}$ & $\begin{array}{l}{ }^{*} \text { PAS } \\
\text { *PAD } \\
{ }_{\downarrow \downarrow}^{+} \text {PAS } \\
{ }^{\star} \text { PAD }\end{array}$ & $\begin{array}{l}{ }^{*} \mathrm{GJ} \\
{ }^{\star} \mathrm{GJ}\end{array}$ \\
\hline CHUNG HK, et al., 2013. & 920 & $\begin{array}{l}0 \text { a } 7 \text { pontos AUDIT } \\
8 \text { a } 15 \text { pontos AUDIT } \\
16 \text { a } 40 \text { pontos AUDIT }\end{array}$ & & $\begin{array}{l}{ }^{*} \mathrm{HDL}-\mathrm{C} \\
{ }^{*} \mathrm{TG} \\
{ }^{\uparrow} \mathrm{HDL}-\mathrm{C} \\
{ }^{\uparrow} \mathrm{TG} \\
{ }^{\uparrow} \mathrm{TDL}-\mathrm{HD} \\
{ }^{\uparrow} \mathrm{TG}\end{array}$ & & $\begin{array}{l}{ }^{*} \mathrm{GJ} \\
{ }^{\star} \mathrm{GJ} \\
{ }^{\uparrow} \mathrm{GJ}\end{array}$ \\
\hline BONNET F, et al., 2012. & 461 & $\begin{array}{c}\text { Abstêmios } \\
<57 \mathrm{~g} / \text { semana } \\
57 \text { a } 130 \mathrm{~g} / \text { semana } \\
>130 \mathrm{~g} / \text { semana }\end{array}$ & $\begin{array}{l}{ }^{*} \mathrm{IMC} \\
{ }^{*} \mathrm{CC} \\
+\mathrm{IMC} \\
+\mathrm{CC} \\
{ }^{\star} \mathrm{IMC} \\
{ }^{+} \mathrm{CC} \\
{ }^{\star} \mathrm{IMC} \\
+\mathrm{CC}\end{array}$ & $\begin{array}{c}{ }^{*} \mathrm{HDL}-\mathrm{C} \\
{ }^{*} \mathrm{TG} \\
{ }^{\uparrow}{ }^{\mathrm{HDL}-\mathrm{HD}} \\
{ }^{\uparrow} \mathrm{TG} \\
{ }^{\uparrow \uparrow} \mathrm{HDL}-\mathrm{HD} \\
{ }^{\uparrow} \mathrm{TG} \\
{ }^{\uparrow \uparrow} \mathrm{HDL}-\mathrm{H} \\
{ }^{+\uparrow} \mathrm{TG}\end{array}$ & $\begin{array}{l}\text { * PAS } \\
\text { *PAD } \\
\text { ^PAS } \\
\text { ^PAD } \\
\text { †PAS } \\
+\mathrm{PAD} \\
\uparrow \mathrm{PAS} \\
\uparrow \mathrm{PAD}\end{array}$ & $\begin{array}{l}{ }^{*} \mathrm{GJ} \\
{ }^{\star G J} \\
+G J \\
+G J\end{array}$ \\
\hline
\end{tabular}




\begin{tabular}{|c|c|c|c|c|c|c|}
\hline \multirow{2}{*}{ Autor } & \multirow{2}{*}{ Amostra } & \multirow{2}{*}{ Variáveis de consumo } & \multicolumn{4}{|c|}{ Parâmetro estudado } \\
\hline & & & Composição corporal & Perfil lipídico & Pressão arterial & Metabolismo glicêmico \\
\hline TERATANI T, et al., 2012. & 8.423 & $\begin{array}{c}\text { 0g/dia } \\
0,1 \text { a } 76,9 g / d i a \\
77,0 \text { a } 153,9 g / d i a \\
154,0 \text { a } 307,9 g / d i a \\
\geq 308,0 \mathrm{~g} / \mathrm{dia}\end{array}$ & & & & $\begin{array}{c}{ }^{\star} \mathrm{DM} 2 \\
\downarrow \mathrm{DM} 2 \\
\downarrow \downarrow \mathrm{DM} 2 \\
\downarrow \downarrow \downarrow \mathrm{DM} 2 \\
\downarrow \downarrow \downarrow \mathrm{DM} 2\end{array}$ \\
\hline $\begin{array}{l}\text { BRANDHAGEN M, et al., } \\
2012 .\end{array}$ & 524 & $\begin{array}{l}\text { Baixa ingestão } \\
\text { Alta ingestão }\end{array}$ & $\begin{array}{l}{ }^{*} \mathrm{IMC} \\
{ }^{*} \mathrm{CC} \\
{ }^{\uparrow} \mathrm{IMC} \\
{ }^{\uparrow} \mathrm{CC}\end{array}$ & & & \\
\hline WAKABAYASHI I., 2012. & 21.572 & $\begin{array}{l}\text { Abstêmios } \\
<22 \mathrm{~g} / \mathrm{dia} \\
22 \text { a } 43 \mathrm{~g} / \mathrm{dia} \\
\geq 44 \mathrm{~g} / \mathrm{dia}\end{array}$ & $\begin{array}{l}{ }^{*} \text { IMC } \\
{ }^{\star} \text { IMC } \\
{ }^{\star} \text { IMC } \\
{ }^{\star} \mathrm{IMC}\end{array}$ & $\begin{array}{c}{ }^{*} \mathrm{HDL}-\mathrm{C} \\
{ }^{*} \mathrm{TG} \\
{ }^{\star} \mathrm{HDL}-\mathrm{C} \\
{ }^{\star} \mathrm{TG} \\
{ }^{\uparrow \uparrow} \mathrm{HDL}-\mathrm{H} \\
{ }^{\uparrow} \mathrm{TG} \\
{ }^{\uparrow \uparrow} \mathrm{TDL}-\mathrm{H} \\
{ }^{\uparrow \uparrow} \mathrm{H} \text { TG }\end{array}$ & & \\
\hline TIMON R, et al., 2012. & 180 & $\begin{array}{l}\text { Não bebedores } \\
1 \text { dia/semana }\end{array}$ & $\begin{array}{l}{ }^{*} \mathrm{IMC} \\
{ }^{*} \mathrm{CC} \\
{ }^{\uparrow} \mathrm{IMC} \\
{ }^{\uparrow} \mathrm{CC}\end{array}$ & $\begin{aligned} & { }^{*} \mathrm{HDL}-\mathrm{C} \\
& * \mathrm{TG} \\
& \\
& \mathrm{HDL}-\mathrm{C} \\
& \uparrow \mathrm{TG}\end{aligned}$ & $\begin{array}{l}\text { *PAS } \\
* \text { PAD } \\
{ }^{\star} \text { PAS } \\
{ }^{+} \text {PAD }\end{array}$ & $\begin{array}{l}{ }^{*} \mathrm{GJ} \\
{ }^{\star} \mathrm{GJ}\end{array}$ \\
\hline & & 2 dias/semana & $\stackrel{\uparrow \uparrow}{{ }^{\uparrow} \mathrm{IMC}}$ & $\begin{array}{l}{ }^{\uparrow} \underset{\uparrow \uparrow \mathrm{HL}-\mathrm{C}}{\mathrm{H}} \mathrm{TG} \\
\end{array}$ & $\begin{array}{c}{ }^{\uparrow \uparrow} \text { PAS } \\
\uparrow \mathrm{PAD}\end{array}$ & ${ }^{\downarrow} \mathrm{GJ}$ \\
\hline ROH W. et al., 2009. & 1.717 & $\begin{array}{c}\text { 0g/dia } \\
1 \text { a } 14 \mathrm{~g} / \mathrm{dia} \\
15 \text { a } 29 \mathrm{~g} / \mathrm{dia} \\
\geq 30 \mathrm{~g} / \mathrm{dia}\end{array}$ & & & $\begin{array}{l}\text { * PAS } \\
\text { * PAD } \\
\text { tPAS } \\
\text { tPAD } \\
{ }^{\star} \text { PAS } \\
{ }^{\uparrow} \mathrm{PAD} \\
{ }^{\uparrow} \mathrm{PAS} \\
{ }^{\uparrow} \mathrm{PAD}\end{array}$ & $\begin{array}{c}{ }^{*} \mathrm{GJ} \\
{ }^{*} \mathrm{DM} 2 \\
+\mathrm{GJ} \\
+\mathrm{DM} 2 \\
{ }^{\uparrow} \mathrm{GJ} \\
{ }^{\uparrow} \mathrm{DM} 2 \\
{ }^{\uparrow \uparrow} \mathrm{GJ} \\
{ }^{\uparrow} \mathrm{DM} 2\end{array}$ \\
\hline
\end{tabular}

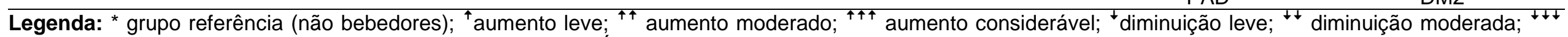

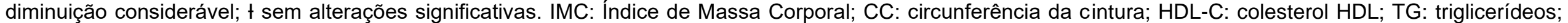

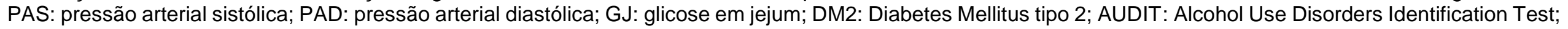
RDA: Recommended Dietary Allowances.

Fonte: Klier A, et al., 2020. 
Percebe-se que a maioria dos estudos foca em apenas um ou dois componentes da SM, apenas 3 observam todos os parâmetros de interesse do estudo (TIMON R, et al., 2012; BONNET F, et al., 2012; ANEJA J, et al., 2013). Além disso, após a leitura detalhada dos manuscritos, foi possível observar que o álcool impacta de forma distinta nos parâmetros da SM em homens e mulheres, sendo assim, os estudos foram classificados de acordo com esta perspectiva (Tabela 2, Tabela 3, Tabela 4).

Ressalta-se que, em relação à população masculina, os impactos negativos do consumo de álcool nos parâmetros da SM estão relacionados ao perfil lipídico, ou seja, alterações representativas no HDL e triglicerídeos. E quanto aos positivos, não foi possível observar.

Tabela 3 - Apresentação dos resultados dos artigos que estudaram a população feminina, incluindo autor, amostra e variáveis de consumo alcoólico.

\begin{tabular}{|c|c|c|c|c|c|c|}
\hline \multirow[b]{2}{*}{ Autor } & \multirow[b]{2}{*}{ Amostra } & \multirow[b]{2}{*}{$\begin{array}{l}\text { Variáveis de } \\
\text { consumo }\end{array}$} & \multicolumn{4}{|c|}{ Parâmetro estudado } \\
\hline & & & $\begin{array}{c}\text { Composição } \\
\text { corporal }\end{array}$ & $\begin{array}{l}\text { Perfil } \\
\text { lipídico }\end{array}$ & $\begin{array}{c}\text { Pressão } \\
\text { arterial }\end{array}$ & $\begin{array}{c}\text { Metabolismo } \\
\text { glicêmico }\end{array}$ \\
\hline \multirow{3}{*}{$\begin{array}{l}\text { ROSOFF DB, et } \\
\text { al., } 2019 .\end{array}$} & \multirow{3}{*}{541} & $<4$ doses/dia & & $\begin{array}{l}{ }^{*} \mathrm{HDL}-\mathrm{C} \\
{ }^{*} \mathrm{TG}\end{array}$ & & \\
\hline & & $\begin{array}{c}4 \text { a } 7 \\
\text { doses/dia } \\
8 \text { a } 11 \\
\text { doses/dia }\end{array}$ & & $\begin{array}{c}{ }^{\uparrow} \mathrm{HDL}-\mathrm{C} \\
{ }_{\uparrow \uparrow \uparrow} \mathrm{TG} \\
{ }^{\uparrow} \mathrm{HDL}-\mathrm{C} \\
{ }^{\uparrow} \uparrow \uparrow \mathrm{TG}\end{array}$ & & \\
\hline & & $\geq 12$ doses dia & & $\begin{array}{c}{ }^{\uparrow \uparrow} \mathrm{HDL}-\mathrm{C} \\
{ }_{\uparrow \uparrow \uparrow} \mathrm{TG}\end{array}$ & & \\
\hline \multirow{5}{*}{$\begin{array}{l}\text { ALBANI V, et } \\
\text { al., } 2018 .\end{array}$} & \multirow{5}{*}{4.967} & \multirow{5}{*}{$\begin{array}{c}0 \% \text { RDA } \\
>0-25 \% \text { RDA } \\
>25-50 \% \\
\text { RDA } \\
>50-75 \% \\
\text { RDA } \\
>75 \% \text { RDA }\end{array}$} & * IMC & & & \\
\hline & & & + IMC & & & \\
\hline & & & + IMC & & & \\
\hline & & & ${ }^{\uparrow} \mathrm{IMC}$ & & & \\
\hline & & & ${ }^{\uparrow} \mathrm{IMC}$ & & & \\
\hline \multirow{5}{*}{$\begin{array}{l}\text { TAYIE FA, et } \\
\text { al., } 2016 .\end{array}$} & \multirow{5}{*}{3.188} & 0 doses/dia & * IMC & & & \\
\hline & & 1 dose/dia & $\downarrow$ IMC & & & \\
\hline & & 2 doses/dia & $\downarrow \downarrow$ IMC & & & \\
\hline & & 3 doses/dia & ${ }^{\downarrow}$ IMC & & & \\
\hline & & $\geq 4$ doses/dia & $\downarrow$ IMC & & & \\
\hline \multirow{4}{*}{$\begin{array}{l}\text { BONNET F, et } \\
\text { al., } 2012 .\end{array}$} & \multirow{4}{*}{563} & Abstêmias & $\begin{array}{l}{ }^{*} \mathrm{IMC} \\
{ }^{*} \mathrm{CC}\end{array}$ & $\begin{array}{c}{ }^{*} \mathrm{HDL}-\mathrm{C} \\
{ }^{*} \mathrm{TG}\end{array}$ & $\begin{array}{l}\text { * PAS } \\
\text { * PAD }\end{array}$ & ${ }^{*} \mathrm{GJ}$ \\
\hline & & $<28 \mathrm{~g} /$ semana & $\begin{array}{l}{ }^{\downarrow} \mathrm{IMC} \\
{ }^{+} \mathrm{CC}\end{array}$ & $\begin{array}{l}{ }^{\uparrow} \mathrm{HDL}-\mathrm{C} \\
+\mathrm{TG}\end{array}$ & $\begin{array}{l}\downarrow \text { PAS } \\
\downarrow \text { PAD }\end{array}$ & $+\mathrm{GJ}$ \\
\hline & & $\begin{array}{c}28 \mathrm{a} \\
64 \mathrm{~g} / \mathrm{semana}\end{array}$ & $\begin{array}{c}\downarrow \downarrow \\
\downarrow \downarrow\end{array}$ & $\begin{array}{c}{ }^{\uparrow \uparrow} \text { HDL-C } \\
\text { †TG }\end{array}$ & $\begin{array}{l}\downarrow \downarrow \text { PAS } \\
\downarrow \downarrow \text { PAD }\end{array}$ & † GJ \\
\hline & & $>64 \mathrm{~g} /$ semana & $\begin{array}{c}\downarrow \downarrow \downarrow \\
\downarrow \downarrow \downarrow\end{array}$ & $\begin{array}{c}{ }^{\uparrow \uparrow} \mathrm{HDL}-\mathrm{C} \\
+\mathrm{TG}\end{array}$ & $\begin{array}{l}\downarrow \text { PAS } \\
\downarrow \text { PAD }\end{array}$ & tGJ \\
\hline \multirow{3}{*}{$\begin{array}{l}\text { BRANDHAGEN } \\
\text { M, et al., } 2012 .\end{array}$} & \multirow{3}{*}{611} & Baixa ingestão & ${ }^{*}$ IMC & & & \\
\hline & & & $+\mathrm{IMC}$ & & & \\
\hline & & Alta ingestão & $+\mathrm{CC}$ & & & \\
\hline \multirow{5}{*}{$\begin{array}{l}\text { DE JESUS } \\
\text { SILVA AB, et } \\
\text { al., } 2011 .\end{array}$} & \multirow{5}{*}{178} & Não bebem & $\begin{array}{l}{ }^{*} \mathrm{IMC} \\
{ }^{*} \mathrm{CC}\end{array}$ & & & \\
\hline & & $<8$ pontos & $\uparrow$ IMC & & & \\
\hline & & AUDIT & ${ }^{\uparrow} \mathrm{CC}$ & & & \\
\hline & & $\geq 8$ pontos & ${ }^{\uparrow \uparrow} \mathrm{IMC}$ & & & \\
\hline & & AUDIT & ${ }^{\uparrow \uparrow} \mathrm{CC}$ & & & \\
\hline
\end{tabular}

cogenda: ${ }^{*}$ grupo referével; ${ }^{\downarrow}$ diminuição leve; ${ }^{\downarrow} \downarrow$ diminuição moderada; $+\downarrow \downarrow$ diminuição considerável; $\downarrow$ sem alterações significativas. IMC: Índice de Massa Corporal; CC: circunferência da cintura; HDL-C: colesterol HDL; TG: triglicerídeos; PAS: pressão arterial sistólica; PAD: pressão arterial diastólica; GJ: glicose em jejum; AUDIT: Alcohol Use Disorders Identification Test; RDA: Recommended Dietary Allowances.

Fonte: Klier A, et al., 2020. 
No que se refere ao público feminino, as pesquisas indicaram impactos negativos similares ao sexo masculino, ou seja, alterações no HDL e triglicerídeos. Aspecto positivo da ingestão do álcool foi relatado em apenas um estudo, em que Bonnet $F$, et al. (2012) relatam a diminuição nos níveis pressóricos.

Tabela 4 - Apresentação dos resultados dos artigos que estudaram a população em geral, sem distinção por sexo, incluindo autor, amostra e variáveis de consumo alcoólico.

\begin{tabular}{|c|c|c|c|c|c|c|}
\hline \multirow[b]{2}{*}{ Autor } & \multirow[b]{2}{*}{ Amostra } & \multirow[b]{2}{*}{$\begin{array}{l}\text { Variáveis de } \\
\text { consumo }\end{array}$} & \multicolumn{4}{|c|}{ Parâmetro estudado } \\
\hline & & & $\begin{array}{c}\text { Composição } \\
\text { corporal }\end{array}$ & $\begin{array}{c}\text { Perfil } \\
\text { lipídico }\end{array}$ & $\begin{array}{c}\text { Pressão } \\
\text { arterial }\end{array}$ & $\begin{array}{c}\text { Metabolismo } \\
\text { glicêmico }\end{array}$ \\
\hline $\begin{array}{l}\text { WELLMAN RJ, } \\
\text { et al., } 2016 .\end{array}$ & 736 & $\begin{array}{c}\text { Nunca } \\
<1 \mathrm{x} / \mathrm{mês} \\
1 \mathrm{a} 3 \mathrm{x} / \mathrm{mês} \\
1 \text { a } 6 \mathrm{x} / \mathrm{semana} \\
\text { Todos os dias }\end{array}$ & & & $\begin{array}{l}{ }^{\star} \text { PAS } \\
{ }^{+} \text {PAS } \\
\text { †PAS } \\
{ }_{\uparrow \uparrow} \text { PAS } \\
{ }^{\uparrow \uparrow} \text { PAS }\end{array}$ & \\
\hline $\begin{array}{l}\text { ABRAMSON J, } \\
\text { et al., } 2009 .\end{array}$ & 157 & $\begin{array}{c}0 \text { doses/semana } \\
1 \text { a } 2 \\
\text { doses/semana } \\
\geq 3 \\
\text { doses/semana }\end{array}$ & & & $\begin{array}{l}\text { * PAS } \\
\text { * PAD } \\
\text { tPAS } \\
\text { ^PAD } \\
\text { ^PAS } \\
{ }^{\uparrow} \text { PAD }\end{array}$ & \\
\hline
\end{tabular}

Legenda: * grupo referência (não bebedores); ${ }^{\uparrow}$ aumento leve; ${ }^{\uparrow \uparrow}$ aumento moderado; ${ }^{\uparrow \uparrow}$ aumento considerável; ${ }^{\downarrow}$ diminuição leve; ${ }^{\downarrow \downarrow}$ diminuição moderada; ${ }^{\downarrow \downarrow \downarrow}$ diminuição considerável; $\downarrow$ sem alterações significativas. PAS: pressão arterial sistólica; PAD: pressão arterial diastólica.

Fonte: Klier A, et al., 2020.

Nos estudos com o público em geral, sem distinção de gênero, o parâmetro observado foi a pressão arterial. Nesses, o álcool impactou negativamente nos níveis pressóricos, gerando um aumento dos mesmos quanto maior fosse a frequência do consumo.

\section{Consumo Alcoólico}

Os estudos apresentados nesta revisão integrativa basearam-se no consumo autorrelatado de álcool, ou seja, os participantes, através de questionários, mensuravam a quantidade, frequência e tipo de bebida consumida. Foram utilizados questionários amplamente conhecidos na atenção primária, como o AUDIT e o Timeline Followback (TLFB), além de outros desenvolvidos especificamente para cada pesquisa. Veja no gráfico abaixo as variáveis de consumo citadas (Gráfico 1).

Gráfico 1 - Variáveis de consumo alcoólico utilizadas nos estudos incluídos nesta revisão integrativa.

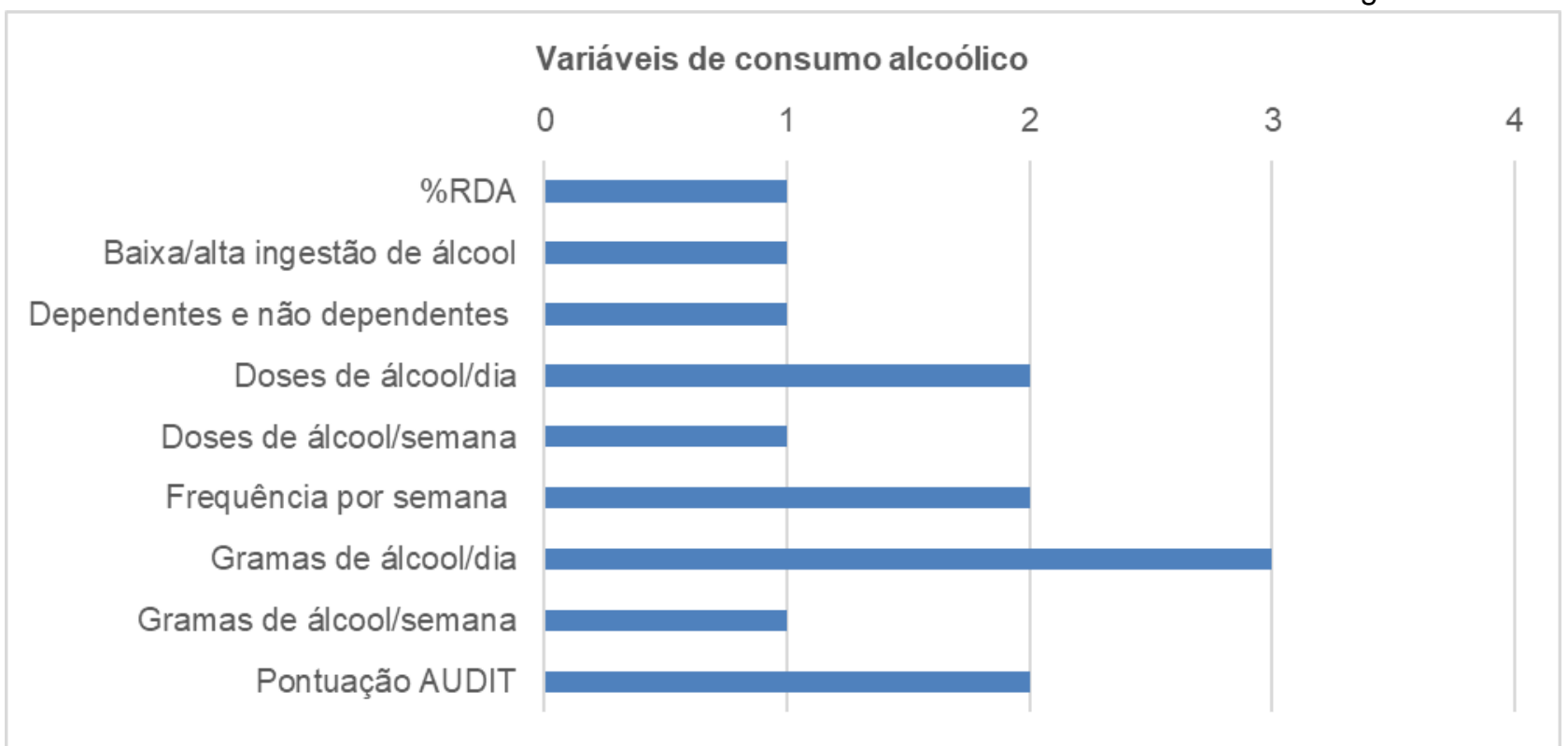

Fonte: Klier A, et al., 2020. 


\section{Composição corporal}

O IMC e CC dos participantes foram avaliados em 8 estudos. Albani V, et al. (2018) conduziram um estudo com 3.838 homens e 4.967 mulheres do Reino Unido, valendo-se de dados de duas pesquisas populacionais, a Health Survey for England (HSE) e a Scotish Health Survey (SHeS). Buscou-se determinar a relação entre a energia consumida pelos indivíduos a partir de bebidas alcoólicas e seu IMC, e, para tanto, a energia foi mensurada como \%RDA. Concluiu-se que homens com ingestão de álcool superior a $75 \%$ RDA e mulheres, a partir de 50\%RDA, apresentam aumento nos valores de IMC.

A pesquisa de Tayie FA, et al. (2016) foi outro estudo que avaliou a associação entre ingestão de álcool e valores de IMC em 3.029 homens e 3.188 mulheres, porém apresenta resultados diferentes. Para os homens, consumir mais do que duas doses de álcool por dia aumentou os valores de IMC, enquanto que qualquer quantidade consumida de álcool associou-se a um IMC menor em mulheres. Este estudo utilizou dados da Pesquisa Nacional de Saúde e Nutrição dos Estados Unidos da América 2007-2010 (2010).

Anteriormente, Bonnet F, et al. (2012), também haviam estudado 461 homens e 563 mulheres, observando a ingestão de álcool em gramas por semana, através de dados do estudo Relação entre Sensibilidade à Insulina e Doença Cardiovascular (RISC) e seu impacto na composição corporal dos participantes. Naquele momento em que a pesquisa ocorreu, identificaram que quanto maior o consumo de álcool, menores os valores de IMC e CC em mulheres, enquanto que, para os homens, apenas valores mais elevados de ingestão alcoólica, impactaram em pequenas quedas de valores de IMC e CC.

A diferença entre a resposta ao álcool de acordo com o gênero também foi estudada por Brandhagen $M$, et al. (2012), analisando dados de 524 homens e 611 mulheres, candidatos à cirurgia bariátrica, participantes do Swedish Obese Subject (SOS), divididos apenas em alto e baixo consumo alcoólico. Os resultados mostraram associação negativa entre o consumo de álcool e o IMC em mulheres, porém associação positiva entre álcool e IMC e CC em homens.

Aneja J, et al. (2013) estudaram a composição corporal de homens, divididos em dependentes de álcool $(n=100)$ e não dependentes ( $n=100)$, de acordo com o diagnóstico CID-10. Os homens foram avaliados no Centro de Tratamento e Desintoxicação de Drogas (DDTC) em Chandigarh, Índia, e, pode-se observar que a dependência de álcool estava associada a uma leve diminuição nos valores de CC e IMC.

Wakabayashi I (2012), também estudou a população masculina ( $n=21.572)$ japonesa, utilizando dados de consultas e exames médicos de rotina nos locais de trabalho, através de questionários específicos. Qualquer consumo de álcool foi associado à diminuição nos valores de IMC, porém, o consumo leve foi mais impactante que o moderado ou pesado. A composição corporal de 180 homens também foi estudada por Timon $\mathrm{R}$, et al. (2012), porém observando em quantos dias de final de semana os jovens espanhóis consumiam álcool, ou seja, em apenas 1 ou nos 2 dias. Concluiu-se que os valores de IMC e CC aumentavam de acordo com a frequência de consumo alcoólico.

O estudo conduzido por De Jesus Silva AB, et al. (2011) avaliou a associação entre a pontuação AUDIT e os valores de IMC e CC de 178 jovens universitárias do curso de Nutrição da Universidade Federal de Ouro Preto e foi capaz de identificar valores dose-resposta positivos de acordo com a pontuação obtida no teste.

Assim, no que se refere à composição corporal, os estudos analisados indicaram, no gênero feminino, tendência de leve redução, porém os estudos não foram unânimes nessa diminuição. Quanto ao sexo masculino, as pesquisas foram inconclusivas.

\section{Perfil lipídico}

Em relação ao perfil lipídico, 6 estudos incluídos nesta revisão integrativa avaliaram TG e HDL-C. Rosoff DB, et al. (2019) investigaram se havia alterações nos níveis de lipídios e enzimas de função hepática circulantes de acordo com a ingestão alcoólica (doses diárias), avaliando 1.519 indivíduos (978 homens e 541 mulheres), e perceberam que estes níveis estavam positivamente associados à quantidade de álcool ingerida, em ambos os sexos. O estudo avaliou a ingestão a partir de dados coletados pelo Instituto Nacional de Abuso de Álcool e Alcoolismo (NIAAA), através do questionário e entrevista TLFB. 
Bonnet $F$, et al. (2012), também estudaram a associação entre ingestão alcoólica e perfil lipídico em 461 homens e 563 mulheres, utilizando dados do estudo Relação entre Sensibilidade à Insulina e Doença Cardiovascular (RISC) e, neste caso, também foi possível observar um incremento nos valores de HDL-C em ambos os sexos, entretanto, os valores de TG em homens demonstraram aumento constante, enquanto que, para as mulheres, ingerir até $28 \mathrm{~g}$ de álcool por semana aumentou o TG, mas ingerir mais que $64 \mathrm{~g}$ por semana o fez diminuir.

Aneja J, et al. (2013) estudaram uma população de 200 homens, divididos em dependentes de álcool $(n=100)$ e não dependentes $(n=100)$, de acordo com o diagnóstico CID-10. A pesquisa ocorreu no Centro de Tratamento e Desintoxicação de Drogas (DDTC) em Chandigarh, Índia, e, através dela, foi possível identificar uma concentração mais elevada de HDL-C entre os dependentes de álcool. Também em 2013, um estudo conduzido na Coreia, utilizando dados da Korea National Health and Nutrition Examination Survey V-1 (KNHANES V-1), avaliou 920 homens e observou que valores de HDL-C e TG se elevavam de acordo com o aumento da pontuação no AUDIT dos indivíduos (CHUNG HK, et al., 2013).

Outro estudo desenvolvido com 21.572 homens japoneses corrobora com esses dados de aumento de HDL-C e TG de acordo com a dose-resposta (WAKABAYASHI I, 2012). Por fim, Timon R, et al. (2012) acompanharam o perfil lipídico de 180 homens espanhóis, que consumiam álcool em um ou dois dias de final de semana e confirmaram a tendência do aumento dos valores de HDL-C e TG.

Assim, no que se refere ao perfil lipídico, os estudos foram unânimes em indicar o impacto negativo, aumentando os índices de HDL-C e TG, independentemente da quantidade/frequência de consumo. Estudos revelam que, em grandes quantidades, o HDL-C pode tornar-se disfuncional, levando a um maior risco de mortalidade por todas as causas e de desenvolvimento de doenças infecciosas, principalmente quando 0 valor é $\geq 100 \mathrm{mg} / \mathrm{dL}$ (MADSEN CM, et al., 2017a; 2017b).

\section{Pressão arterial}

A pressão arterial foi abordada em 6 artigos desta revisão integrativa. Homens ( $n=461)$ e mulheres $(n=563)$ tiveram seus dados do estudo Relação entre Sensibilidade à Insulina e Doença Cardiovascular (RISC) coletados por Bonnet $\mathrm{F}$, et al. (2012), que avaliaram a relação entre a ingestão de álcool em gramas por semana e os níveis pressóricos arteriais, com resultados bastante divergentes. Para os homens, consumir de 0 a $57 \mathrm{~g}$ de álcool por semana e mais que $130 \mathrm{~g}$, foi capaz de aumentar levemente a PAS e PAD, porém quantidades intermediárias não impactaram nesses valores. Com as mulheres, a ingestão de álcool foi associada a uma diminuição nos valores pressóricos, principalmente quando se consumia entre 28 e $64 \mathrm{~g}$ de álcool por semana.

Este estudo com 200 homens, divididos em dependentes de álcool $(n=100)$ e não dependentes $(n=100)$, de acordo com o diagnóstico CID-10, conduzido no Centro de Tratamento e Desintoxicação de Drogas (DDTC) em Chandigarh, Índia, identificou que a dependência de álcool é capaz de diminuir moderadamente os valores de PAS e aumentar levemente os valores de PAD (ANEJA J, et al., 2013).

Em contrapartida, Roh W, et al. (2009), avaliando os dados de exames e consultas médicas rotineiras de 1.717 trabalhadores obesos coreanos, concluíram que um consumo moderado e elevado de álcool impacta positivamente nos níveis de pressão sanguínea. Desta mesma forma, outro estudo também acompanhou apenas homens $(n=180)$ que consumiam quantidades consideráveis de álcool em um ou dois dias de final de semana e observaram a resposta da pressão sanguínea, concluindo que beber em dois dias de final de semana aumenta significativamente os valores de PAS (TIMON R, et al., 2012).

Estes dois estudos avaliaram a relação entre consumo alcoólico e níveis pressóricos sanguíneos sem fazer distinção por sexo e encontraram resultados parecidos (WELLMAN RJ, et al., 2016; ABRAMSON J, et al., 2009).

O primeiro avaliou 756 jovens participantes do estudo Dependência de Nicotina em Adolescentes e o segundo, observou 157 pessoas afiliadas à Emory University, Atlanta, Georgia. Ambos os estudos verificaram uma associação dose-resposta positiva entre o consumo alcóolico e a pressão sanguínea dos participantes. 
Assim esses seis manuscritos relatam dados inconclusivos quanto aos níveis pressóricos, indicando a necessidade, para esse parâmetro, de estudos de longo prazo.

\section{Metabolismo glicêmico}

Dos 14 artigos incluídos no estudo, 6 avaliaram o metabolismo glicêmico dos participantes. Bonnet $F$, et al. (2012) estudaram a associação entre ingestão alcoólica e a resposta glicêmica em 461 homens e 563 mulheres, através dos dados do estudo Relação entre Sensibilidade à Insulina e Doença Cardiovascular (RISC), e identificaram que nenhuma das categorias de consumo alcoólico, em ambos os sexos, foi capaz de interferir significativamente na GJ ou mesmo na glicemia de 2 horas, inclusive aumentando a sensibilidade periférica à insulina.

No ano de 2012, um estudo conduzido por Teratani T, et al. (2012) avaliou 8.423 homens, todos operários de uma siderúrgica japonesa, valendo-se dos dados registrados a partir de consultas e exames periódicos destes na empresa, buscando verificar a associação entre o consumo de álcool e o desenvolvimento de DM2. Foi possível observar uma relação dose-resposta negativa entre a ingestão alcoólica e a DM2 nestes trabalhadores. Neste mesmo ano, Timon R, et al. (2012), acompanhou 180 jovens espanhóis que bebiam quantidades consideráveis de álcool em um ou dois dias de final de semana e também observaram doseresposta negativa em relação ao consumo alcoólico e a GJ.

Uma população de 200 homens, divididos em dependentes de álcool $(n=100)$ e não dependentes $(n=100)$, de acordo com o diagnóstico CID-10, avaliados no Centro de Tratamento e Desintoxicação de Drogas (DDTC) em Chandigarh, Índia, identificou que a dependência de álcool impacta positivamente nos valores de GJ (ANEJA J, et al., 2013).

Um estudo deste mesmo ano, conduzido na Coreia, utilizando dados da Korea National Health and Nutrition Examination Survey V-1 (KNHANES V-1), avaliou 920 homens, buscando associação entre o consumo alcoólico e função insulínica, tendo como parâmetro sua pontuação no AUDIT. Da mesma forma que o estudo anterior, pode-se perceber que a GJ se eleva de acordo com o aumento da ingestão de álcool (CHUNG HK, et al., 2013).

Corroborando com esses resultados na população masculina, Roh W, et al. (2009), avaliaram os dados de exames e consultas periódicas de 1.717 trabalhadores coreanos obesos, buscando associações entre consumo alcoólico e incidência de GJ elevada e incidência de DM2 e concluíram haver associação doseresposta positiva.

Assim como os níveis pressóricos e composição corporal, os estudos não foram conclusivos no que se refere ao metabolismo glicêmico.

\section{Pontos fortes e limitações da revisão}

Os estudos epidemiológicos observacionais selecionados para esta revisão, sendo transversais e de coorte, foram publicados em revistas de alto prestígio na área da nutrição e saúde, com população sempre superior a 100 indivíduos. Porém, justamente por serem estudos observacionais, não foi possível controlar as variáveis de consumo alcoólico, sendo este, geralmente, autorrelatado, bem como ingestão calórica, horário de consumo, atividade física, nível de estresse, dentre outras. Infere-se que os resultados podem ter sido tão divergentes por falta de um rigor no acompanhamento/controle das referidas variáveis.

\section{CONSIDERAÇÕES FINAIS}

Não estão claros, na literatura analisada nesta revisão integrativa, os impactos da ingestão de álcool sobre os parâmetros da SM. Resultados divergentes são encontrados para IMC, CC, pressão arterial e metabolismo glicêmico, considerando-se ou não o sexo dos participantes. A única associação clara está relacionada ao aumento dos valores de HDL-C e TG, em ambos os sexos, sendo alterados a partir de qualquer quantidade de álcool ingerida. A pesquisa teórica, aqui consolidada, evidencia também a necessidade de novos estudos a fim de aclarar o impacto do álcool sobre os parâmetros da SM. 


\section{REFERÊNCIAS}

1. ABRAMSON JL, et al. Relationship of Self-Reported Alcohol Consumption to Ambulatory Blood Pressure in a Sample of Healthy Adults. American Journal of Hypertension, 2009; 23(9): 994-999.

2. AHIMA RS. Adipose Tissue as an Endocrine Organ. Obesity, 2016; 14(Suppl 5): 242S-249S.

3. ANEJA J, et al. Metabolic Syndrome in alcohol-dependent men: a cross-sectional study. Indian Journal of Psychological Medicine, 2013; 35(2): 190-196.

4. ALBANI V, et al. Examining associations between body mass index in 18-25 year-olds and energy intake from alcohol: Findings from the health survey for England and the scottish health survey. Nutrients, 2018; 10(10): 1477.

5. ARCR - ALCOHOL RESEARCH: CURRENT REVIEWS EDITORIAL STAFF. Drinking patterns and their definitions. Alcohol research: current reviews, 2018; 39(1): 17-18.

6. BONNET F, et al. Moderate alcohol consumption is associated with improved insulin sensitivity, reduced basal insulin secretion rate and lower fasting glucagon concentration in healthy women. Diabetologia, 2012; 55: 3228-3237.

7. BOTELHO LLR, et al. O método da revisão integrativa nos estudos organizacionais. Gestão e Sociedade, 2011; 5(11): 121-136.

8. BRANDHAGEN M, et al. Alcohol and macronutrient intake patterns are related to general and central adiposity. European Journal of Clinical Nutrition, 2012; 66: 305-313.

9. CHUNG HK, et al. Alcohol use behaviors, fat intake and the function of pancreatic $\beta$-cells in non-obese, healthy Korean males: Findings from 2010 Korea national health and nutrition examination survey. Annals of Nutrition and Metabolism, 2013; 62(2): 129-136.

10. DE JESUS SILVA AB, et al. Relação entre consumo de bebidas alcoólicas por universitárias e adiposidade corporal. Jornal Brasileiro de Psiquiatria, 2011; 60(3): 210-215.

11. HUMENIUK R, et al. Self-help strategies for cutting down or stopping substance use: a guide. Geneva: World Health Organization, 2010.

12. MADSEN CM, et al. Extreme high high-density lipoprotein cholesterol is paradoxically associated with high mortality in men and women: two prospective cohort studies. European Heart Journal, 2017a; 38(32): 2478-2486.

13. MADSEN CM, et al. U-shaped relationship of HDL and risk of infectious disease: two prospective population-based cohort studies. European Heart Journal, 2017b; 39(14): 1181-1190.

14. MANTHEY J, et al. Global alcohol exposure between 1990 and 2017 and forecasts until 2030: a modelling study. Lancet, 2019; 6736(18): 1-10.

15. NCEP-ATP III. Expert Panel on Detection, Evaluation, and Treatment of High Blood Cholesterol in Adults (Adult Treatment Panel III). Jama: The Journal of the American Medical Association, 2001; 285(19): 2486-2497.

16. RAMIRES EKNM, et al. Prevalence and factors associated with metabolic syndrome among brazilian adult population: National health survey - 2013. Arquivos Brasileiros de Cardiologia, 2018; 110(5): 455-466.

17. $\mathrm{ROH} \mathrm{W}$, et al. Alcohol consumption and higher incidence of impaired fasting glucose or type 2 diabetes in obese Korean men. Alcohol, 2009; 43(8): 643-648.

18. ROSOFF DB, et al. Association of High-Intensity Binge Drinking With Lipid and Liver Function Enzyme Levels. JAMA network open, 2019; 2(6): e195844.

19. SAKLAYEN MG. The global epidemic of the metabolic syndrome. Current Hypertension Reports, 2018; 20(12): 1-8.

20. SOCIEDADE BRASILEIRA DE CARDIOLOGIA. 1aㅗ Diretriz brasileira de diagnóstico e tratamento da síndrome metabólica. Arq Bras Cardiol, 2005; 84(Supl 1): 1-28.

21. TAYIE FA, et al. Alcoholic beverage consumption contributes to caloric and moisture intakes and body weight status. Nutrition, 2016; 32: 799-805.

22. TERATANI T, et al. Dose-response relationship between tobacco or alcohol consumption and the development of diabetes mellitus in japanese male workers. Drug and Alcohol Dependence, 2012; 125: 276-282.

23. TIMON R, et al. Effects of regular and abusive intake of alcohol at weekends on physiological parameters in Spanish young. Public Health, 2012; 126(10): 873-880.

24. WAKABAYASHI I. Inverse Association Between Triglycerides-to-HDL- Cholesterol Ratio and Alcohol Drinking in Middle-Aged. Journal of Studies on Alcohol and Drugs, 2012; 73(6): 998-1004.

25. WELLMAN RJ, et al. Relationships between Current and Past Binge Drinking and Systolic Blood Pressure in Young Adults. Journal of Adolescent Health, 2016; 58(3): 352-357.

26. $\mathrm{XU} \mathrm{H}$, et al. Etiology of metabolic syndrome and dietary intervention. International Journal of Molecular Sciences, $2019 ; 20(128): 1-19$. 